Article

\title{
A Speculative Model of Thermally Forced Quasi-Geostrophic Flow in an Unbounded Ocean
}

\author{
Fulvio Crisciani ${ }^{1}$, Roberto Purini ${ }^{2}$ and Renzo Mosetti ${ }^{3, *}$ \\ 1 Affiliation 1; Dept. of Physics, University of Trieste, Trieste, Italy ; fulviocrisciani@gmail.com \\ 2 Affiliation 2; ISAC-CNR, Roma, Italy; purrob@libero.it \\ 3 Affiliation 3; OGS-Istituto Nazionale di Oceanografia e di Geofisica Sperimentale, Trieste, Italy; \\ rmosetti@inogs.it \\ * Correspondence: rmosetti@inogs.it
}

Abstract: Starting with a hypothetical geostrophic zonal current in an unbounded ocean, the investigation points out the response of this simple system to a thermal forcing, applied to the free surface and consistent with the maintenance of the geostrophic balance. The main result is the formation of a meridional component of the current, according to the Sverdrup relation, such that the full velocity vector rotates clockwise for heating and anticlockwise for cooling to adjust eventually in the initial zonal direction for large depths.

Keywords: Geophysical Fluid Dynamics, Geostrophic flows; Thermal forcing; Analytical model

\section{Introduction}

In the large-scale ocean circulation the effect of the thermal forcing is prevailingly indirect. It reveals itself essentially through the overlying wind field which is ultimately the response of the atmospere to the horizontal component of the air density gradient due to solar heating. This is the origin of the wind-driven circulation. On the other hand, a direct effect concerns with the heat flux at the air-sea interface, of seasonal or meteorological origin, the latter being able to induce a marked convective circulation on a local scale. Besides the "extreme" cases cited above, in which the forcing has a drastic influence, one can conceive a system in which a less intense heating/cooling at the sea surface makes rise to a weak disturbance superimposed to a hypothetical unperturbed circulation. In this context, most of the existing literature deals with numerically solved high-resolution nodels of circulation in rotating closed basins [1], [2] and papers quoted therein. However, remarkable analytical contributions are found in Barcilon, V., and J. Pedlosky, 1967 [3], Whitehead and Pedlosky ,2000 [4], and Pedlosky (2003) [5]. The aim of this investigation is to explore the interplay between the heating/cooling of a thin sub-superficial layer of an unbounded ocean and the consequent mechanical reaction of the quasi-geostrophic flow field induced by the redistribution of sea-water density inside the water column. The heat flux is weak enough to preserve the dominance of the geostrophic balance in the system. The method resorts (i) to a thermodynamic equation which links together the time rate of heat transfer into/out the ocean surface with the ageostrophic vertical current and (ii) to the Sverdrup relation which connects the vertical current to the meridional transport. In the framework of an analytic approach to above model, only a local solution seems to be accessible. In any case the latter solves exactly the vertical structure of the geostrophic flow and allows to 


$$
\frac{D \rho}{D t}=-\frac{\rho_{0} \alpha_{\theta}}{c_{p}} \dot{Q}
$$
in the initial zonal direction for large depths.

\section{The link between thermal forcing and dynamics}

$$
\frac{\partial \rho}{\partial \theta}=-\rho_{0} \alpha_{\theta}
$$
expansion. Then assume the thermodynamic equation

$$
\frac{D \rho}{D t}=\left(\frac{\partial \rho}{\partial \theta}\right)_{S=S_{0}} \frac{\dot{Q}}{c_{p}}
$$
expressed as (see, for instance, eq. (2.554) of [6])

elucidate the physics of the interplay. The main effect of the thermal forcing on a hypothetical unperturbed westward zonal current is the addition of a small meridional component. In particular, the velocity vector rotates clockwise for heating and anticlochwise for cooling. For increasing depth the current undergoes a further counter-rotation, far smaller than the first one, to adjust eventually

In this section a relationship between the time rate of heat transferred into, or released by, a salinity conserving sea-water volume and the vertical current taking place in the same volume is inferred.

Consider first the linearized equation of state $\rho=\rho(\theta)$, where $\rho$ is the density and $\theta$ is the temperature of sea-water, which yields (see, for instance, eq. (2.17) of [6])

In (1) $\rho_{0}$ is the density around which the equation is linearized and $\alpha_{\theta}$ is the coefficient of thermal

which comes from the first principle for an incompressible fluid (see, for instance, eq. (2.50) of [6]). In (2) $S_{0}$ is the constant value of salinity, $\dot{Q}$ is the time rate of transferred, or released, heat into seawater and $c_{p}$ is its specific heat at constant pressure. Equations (1) and (2) yield

The fundamental role of equation (3) lies in the fact that it includes implicitly the vertical velocity in the advective term of the Lagrangian derivative and, explicitly, the time rate of heat transfer; thus (3) links the dynamics of the flow with the thermal forcing which acts on it. Owing to the prevailing geostrophic and hydrostatic balances governing geophysical flows, the density field is suitably

$$
\rho=\rho_{s}(z)\left[1+r \rho^{\prime}\left(\mathbf{x}^{\prime}, t^{\prime}\right)\right]
$$

In (4) $\rho_{s}$ is the "standard" density, which retains the basic vertical structure of the overall field density while $\rho^{\prime}$ is the non dimensional contribute required by the geostrophic balance. Finally,

$$
r=\frac{U L f_{0}}{g Z} \quad O\left(\frac{U L f_{0}}{g Z}\right) \approx 10^{-4}
$$




$$
\begin{gathered}
(u, v, \rho, p)=\left(u_{0}, v_{0}, \rho_{0}, p_{0}\right)+b\left(u_{1}, v_{1}, \rho_{1}, p_{1}\right)+\ldots \\
w=b w_{1}+\ldots
\end{gathered}
$$

is a small quantity depending on the scales of the motion $L, Z, U^{1}$ and on the planetary constants $f_{0}$ and $g$. Substitution of (4) into (3) produces the desired relationship between the heat and the vertical current (see the Appendix for details) in the non dimensional form (primed variables)

$$
w^{\prime}=\frac{\varepsilon}{S}\left(\frac{D \rho^{\prime}}{D t^{\prime}}+\mathrm{H}^{\prime}\right)
$$

In (6) $w^{\prime}$ is the vertical velocity of the fluid, $\mathcal{E}$ is the Rossby number, $S$ is the stratification parameter (unfortunately the notation is the same as salinity, but the latter will not be used in what follows) and $\mathrm{H}^{\prime}=\frac{g Z \alpha_{\theta} \dot{Q}}{U^{2} f_{0} c_{p}}$ is a non dimensional heating function.

\section{The quasi-geostrophic potential vorticity equation at the oceanic basin scale with thermal forcing}

The quasi-geostrophic dynamics at the oceanic basin scale is widely described, for instance, in [6] and in [7]. Its basic feature is the dominance of planetary vorticity with respect to relative vorticity and this fact makes the formal aspect of the resulting governing equation of potential vorticity noticeably simple. Only the main points, not to be ignored, are recalled below. The non dimensional fields appearing in the primitive equations, that is velocity, density and pressure, are expanded in powers of the ordering parameter

$$
b=\varepsilon \beta
$$

or, alternatively,

$$
b=\beta_{0} L / f_{0}
$$

where $\beta=\beta_{0} L^{2} / U$ and $\beta_{0}$ is the planetary vorticity gradient. At the basin scale

$$
O(b)=10^{-1}
$$

and

$$
O(\beta S)=1
$$

Thus (primes are dropped)

where $u_{0}$ and $v_{0}$ are in geostrophic balance with the pressure gradient $\nabla_{h} p_{0}$ and

\footnotetext{
${ }^{1}$ The depth of the motion is represented by $Z$ while $\mathrm{H}$ means a heating function.
} 
95

$$
\rho_{0}+\frac{\partial p_{0}}{\partial z}=0
$$

96

The first key equation involving two of the fields (10) is the fundamental Sverdrup relation (widely discussed, for instance, in [8])

98

$$
v_{0}=\frac{\partial w_{1}}{\partial z}
$$

which is one of the main ingredients of this investigation. Equation (12) is also the basis of the so

100 called Sverdrup balance of the wind-driven circulation which, however, has nothing to do with this 101 model. The second equation comes from (6) by using (7a) and (10). In fact, substitution of (10) into (6) 102 gives

$$
b w_{1}+\ldots=\frac{\varepsilon}{S}\left(\frac{D_{0}}{D t} \rho_{0}+\mathrm{H}\right)+\ldots
$$

104 where $\frac{D_{0}}{D t}=\frac{\partial}{\partial t}+\mathbf{u}_{0} \cdot \nabla$ and $\mathbf{u}_{0}=\left(u_{0}, v_{0}\right)$. The balance between the 1.h.s. and the r.h.s. of (13),

105 where $O(\mathrm{H})=1$ (see (A11) of the Appendix), is achieved noting that, according to (7a) and to (9),

$$
\frac{\varepsilon}{S}=\frac{\beta \varepsilon}{\beta S}=\frac{b}{\beta S} \text { and } O\left(\frac{b}{\beta S}\right)=O(b) \text {. Thus (13) gives }
$$

$$
w_{1}=\frac{1}{\beta S}\left(\frac{D_{0}}{D t} \rho_{0}+\mathrm{H}\right)
$$

108 Finally, recalling (11), equation (14) becomes

$$
w_{1}=-\frac{1}{\beta S}\left(\frac{D_{0}}{D t} \frac{\partial p_{0}}{\partial z}-\mathrm{H}\right)
$$

110 At this point the vertical velocity $w_{1}$ can be eliminated from (12) and (15) in favour of the sole

111 geostrophic fields to obtain

$$
\frac{\partial}{\partial z}\left\lfloor\frac{1}{\beta S}\left(\frac{D_{0}}{D t} \frac{\partial p_{0}}{\partial z}-\mathrm{H}\right)\right\rfloor+v_{0}=0
$$

113 The steady version of (16) written by means of the streamfunction $\psi=p_{0}$, in terms of which

$114 \frac{D_{0}}{D t} \frac{\partial p_{0}}{\partial z}=J\left(\psi, \frac{\partial \psi}{\partial z}\right)$ and $v_{0}=\frac{\partial \psi}{\partial x}$, takes the form

$$
\frac{\partial}{\partial z}\left\{\frac{1}{\beta S}\left[J\left(\psi, \frac{\partial \psi}{\partial z}\right)-\mathrm{H}\right]\right\}+\frac{\partial \psi}{\partial x}=0
$$


116 By resorting to the same notation as in (17), equation (15) becomes

$$
w_{1}=-\frac{1}{\beta S}\left[J\left(\psi, \frac{\partial \psi}{\partial z}\right)-\mathrm{H}\right]
$$

118 Equation (18) will be used to express the vertical boundary condition of the model solution in $Z=0$

119 by means of $\psi$ under the assumption of the rigid lid approximation, in accordance with [7].

\section{4. The Model}

121 The model aims to focus the vertical structure of the geostrophic flow by means of suitable 122 simplifications whose validity is essentially local. The system is steady and governed by a special 123 version of (17) under the following hypotheses:

- The fluid is vertically included in the interval $(-\infty<z \leq 0)$;

- $\mathrm{H}=\mathrm{H}(z), \mathrm{O}(\mathrm{H})=1, \mathrm{H}(-\infty)=0$;

- The vertical boundary condition $w_{1}=0$ in $z=0$ is given by (18)

- At large depths the current is mainly zonal

$$
J\left(\psi, \frac{\partial \psi}{\partial z}\right)_{z=0}=\mathrm{H}(0)
$$

$$
\psi(x, y,-\infty)=\psi_{0}(y)
$$

Thus, equation (17) simplifies into

133 and the model is summarized in equations (19), (20), (21). The thermal forcing can be conceived as a

134 disturbance of the zonal current $u_{0}=-\frac{d \psi_{0}}{d y}$, where $\psi=\psi_{0}(y)$, which identically satisfies the 135 unperturbed motion (i.e., the motion in the absence of $\mathrm{H}$ ) on the beta-plane. Hereafter, for 136 simplicity, the constant westward current $u_{0}=-1$ will be considered, so

$$
\frac{\partial}{\partial z} J\left(\psi, \frac{\partial \psi}{\partial z}\right)+\beta S \frac{\partial \psi}{\partial x}=\frac{d \mathrm{H}}{d z}
$$

$138 \bar{y}$ being a constant. The effect of the forcing is expected to introduce a baroclinic term $\phi(x, y, z)$ 139 superimposed to (22), that is to say

$$
\psi=y-\bar{y}+\phi(x, y, z)
$$


141

142

Substitution of (23) into (21) yields

143

144

Because the r.h.s. of (24) is a function of $Z$ alone, it seems sensible to try a solution of the form

$$
\phi=(x-\bar{x}) \varphi(z)
$$

where the constant $\bar{X}$ can be identified with the longitude of the eastern boundary of the fluid domain. Thus $X-\bar{X} \leq 0$. Owing to (25), the Jacobian determinant of (24) is identically zero and the

$$
\beta S \varphi-\frac{d^{2} \varphi}{d z^{2}}=\frac{d \mathrm{H}}{d z}
$$

In turn, substitution of the full streamfunction (23) with (25) into (19) gives the boundary condition

$$
\left(\frac{d \varphi}{d z}\right)_{z=0}=-\mathrm{H}(0)
$$

while boundary condition (20) implies

$$
\varphi(-\infty)=0
$$

154 Trivially $\nabla_{h}^{2} \psi=\nabla_{h}^{2}[y-\bar{y}+(x-\bar{x}) \varphi(z)]=0$, so the request that, at the basin scale, relative 155 vorticity be far smaller than planetary vorticity is satisfied a fortiori. Problem (26), (27), (28) can be 156 solved once $\mathrm{H}(z)$ is given. The hypothesis

$$
\mathrm{H}(z)=\mathrm{H}_{0} \exp (\lambda z)
$$

158 is hereafter taken into account, where $O\left(\mathrm{H}_{0}\right)=1$ and $\lambda>>1$. A wide variety of functions could 159 be considered in place of (29), however the latter seems to be preferable because of its simple form 160 and because it is fit for describing the absorption process $\mathrm{H}(z-d z)=-\lambda \mathrm{H}(z) d z$ into sea water

$161(z \leq 0)$. Heating implies $\mathrm{H}_{0}>0$ while cooling $\mathrm{H}_{0}<0$. Moreover, setting $Z_{*}=Z Z$, the quantity $162 \lambda Z=\frac{Z_{*}}{Z / \lambda}$ appearing in (29) introduces the typical heating/cooling depth $Z / \lambda<<Z$. Substitution 163 of (29) into (26) yields

$$
\beta S \varphi-\frac{d^{2} \varphi}{d z^{2}}=\lambda \mathrm{H}_{0} \exp (\lambda z)
$$




$$
\varphi(z)=\frac{\mathrm{H}_{0}}{\beta S-\lambda^{2}}[\lambda \exp (\lambda z)-\sqrt{\beta S} \exp (\sqrt{\beta S} z)]
$$

where $\beta S-\lambda^{2}<<1$. Solution (31) allows to evaluate all the geostrophic fields, that is to say

$$
u_{0}=-1, v_{0}=\varphi(z), p_{0}=y-\bar{y}+(x-\bar{x}) \varphi(z), \rho_{0}=-(x-\bar{x}) \frac{d \varphi}{d z}
$$

169 Note that $\rho_{0}$ has the same sign as $\frac{d \varphi}{d z}$. In turn, substitution of (31) into (18) gives the ageostrophic 170 vertical velocity

$$
w_{1}=\frac{\mathrm{H}_{0}}{\beta S-\lambda^{2}}[\exp (\lambda z)-\exp (\sqrt{\beta S} z)]
$$

172 One can check that (31) and (32) identically satisfy equation (12). From (31), one finds $\int_{-\infty}^{0} \varphi(z) d z=0$

173 , in accordance with the relationships $w_{1}(0)=w_{1}(-\infty)=0$ that follow from (32). In particular $174 \varphi(0)=-\frac{\mathrm{H}_{0}}{\sqrt{\beta S}+\lambda} ;$ moreover $\varphi$ changes its sign, only once, at the depth

$$
z_{0}=\frac{1}{\sqrt{\beta S}-\lambda} \ln \frac{\lambda}{\sqrt{\beta S}}
$$

176 Therefore, in the presence of heating, $\varphi$ is negative (i.e., $v_{0}$ southward) from the free surface 177 down to $Z_{0}$ and positive (i.e., $v_{0}$ northward) below $Z_{0}$. Because of the inequalities $178 \exp (\lambda z) \leq \exp (\sqrt{\beta S} z) \forall z \in(0,-\infty)$ and $\beta S-\lambda^{2}<0$, the vertical velocity (32) has the same 179 sign as $\mathrm{H}_{0}$ in the full water column (upward for heating and downward for cooling) and reaches

180 its extremum in (33), where the meridional velocity changes its sign. The density anomaly $\rho_{0}$ is 181 negative for heating and positive for cooling in the layer from the free surface down to the depth $182 z=2 z_{0}$. Although it changes its sign below $z=2 z_{0}$, the total density anomaly $183 \int_{-\infty}^{0} \rho_{0} d x=(x-\bar{x}) \frac{\mathrm{H}_{0}}{\sqrt{\beta S}+\lambda}$ retains a sign opposite to that of $\mathrm{H}_{0}$ as in the upper layer $184\left(2 z_{0}<z \leq 0\right)$. All this summarizes the dynamics of the thermal forcing. In fact heating squizees the 
185

186

187

188

189

190

191

194

195

196

198

201

To visualize a definite solution, the following values

$$
\beta S=1, \mathrm{H}_{0}=1, \lambda=10
$$

are hereafter fixed. Therefore

$$
v_{0}(z)=\frac{1}{99}[\exp (z)-10 \exp (10 z)]
$$

$$
w_{1}(z)=\frac{1}{99}[\exp (z)-\exp (10 z)]
$$
upper part of the column where a direct computation yields

$$
M_{y}=-w_{1}\left(z=z_{0}\right)
$$
to the semi-axis $x>0$, anticlockwise oriented. Openly

$$
\tan [\delta(z)]=-\varphi(z)
$$
according to the identity $\tan (\delta)=\tan (\delta-\pi)$, the inversion of (35) gives

$$
\delta(z)=\pi-\tan ^{-1}[\varphi(z)]
$$

upper portion of the fluid column and stretches the remaining part in accordance with the upward

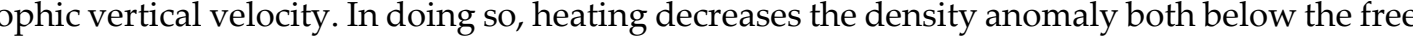
meridional southward transport $M_{y}=\int_{z_{0}}^{0} v_{0} d z$ with a negative density anomaly takes place in the

while the meridional northward transport equal to $-M_{y}$ forms in the remaining part. The dynamics is symmetrically reverted for cooling. A further noticeable quantity describing the structure of the flow field is the angle, say $\delta$, singled out, at each depth, by the vector $\mathbf{u}_{0}=-\hat{\mathbf{i}}+\varphi(z) \hat{\mathbf{j}}$ with respect

where the periodicity $\pi$ of the tangent function must be taken into account in the inversion of (35). In fact, the geostrophic current at large depth $\mathbf{u}_{0}(z=-\infty)=-\hat{\mathbf{i}}$ implies $\delta(-\infty)=\pi$ and therefore, 
(a) $\mathrm{v}$

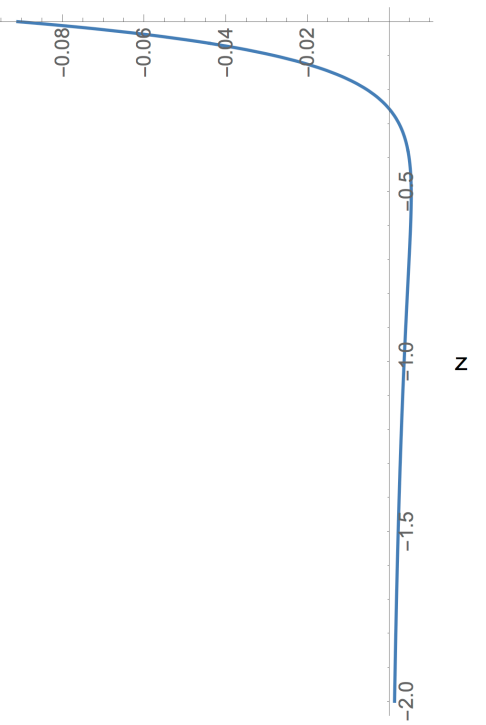

(c) $\rho / \Delta \mathbf{x}$

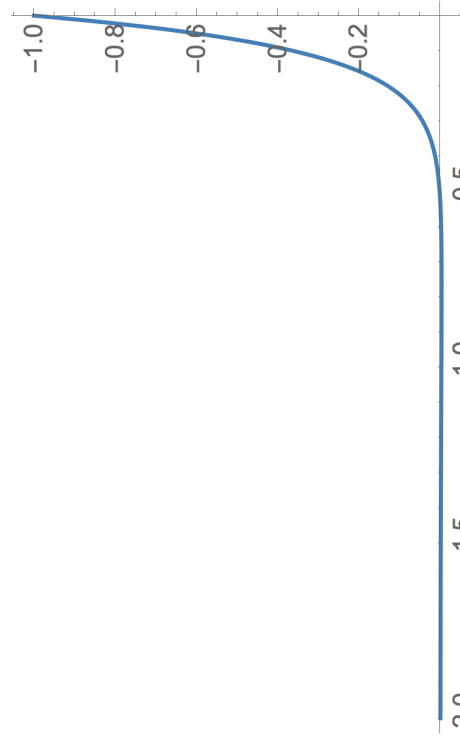

(b) w

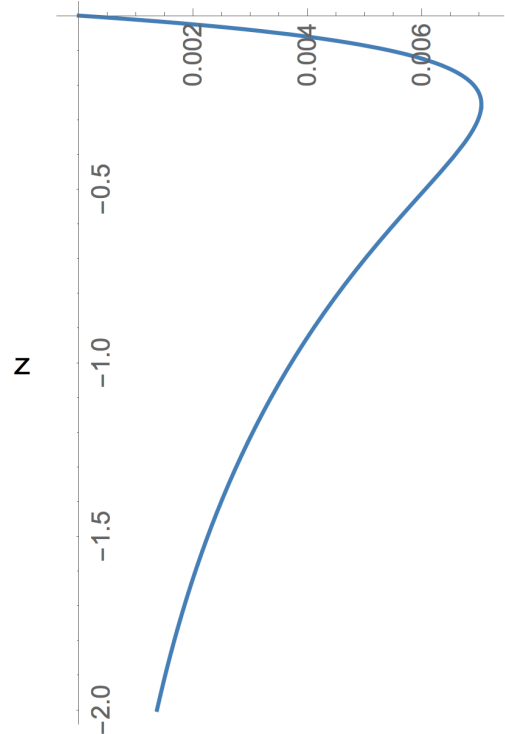

(d) $\delta$

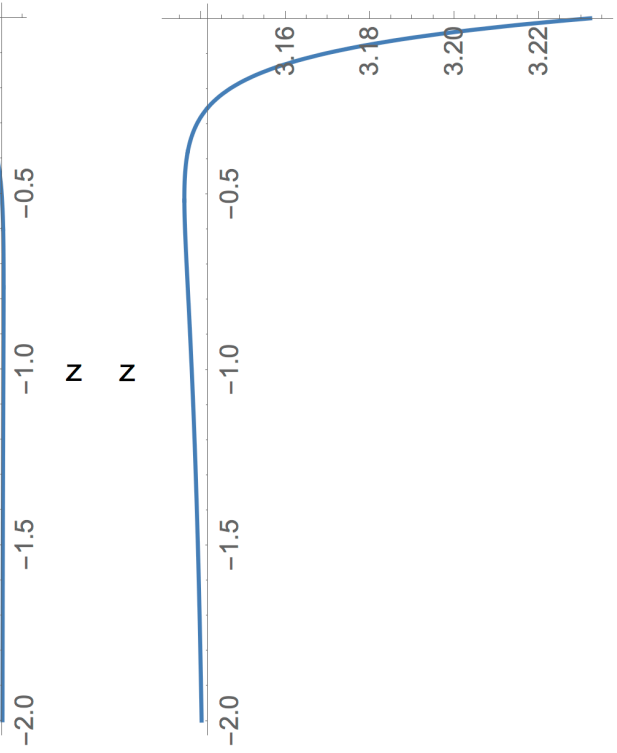

212 Figure 1. Panel (a) Vertical profile of the meridional geostrophic current in the presence of heating;

213 Panel (b) Vertical profile of the a-geostrophic vertical velocity; Panel (c) Vertical profile of the ratio

$214 \rho_{0} /(\overline{\mathrm{x}}-\mathrm{x})$; Panel (d) Vertical profile of the angle $\delta$ between the velocity vector and the negative

$215 \mathrm{x}$-axis, evaluated counter-clockwise. Note the small counter-clockwise rotation below $\mathrm{Z}_{0}$.

216 Panel (a) points out the inversion of the meridional velocity around the depth $z_{0} \approx-0.256$, 217 from southward to northward. At the same depth the a-geostrophic vertical velocity reaches its 218 maximum, according to panel (b). For every fixed longitude $X$, the density anomaly induced by 219 heating is almost exactly negative as one can ascertain from panel (c). Finally, the anticlockwise 220 rotation of the velocity vector $\mathbf{u}_{0}$ is made evident in panel (d): in particular, the right zonal direction

221 takes place both at $Z=Z_{0}$ and at great depths, in accordance with (28). Note the small counter- 
222 rotation of $\mathbf{u}_{0}$ below $Z_{0}$. The vertical motion of a parcel, say $Z=Z(t)$, can be singled out by solving

223 the problem

$$
\left\{\begin{array}{c}
\dot{z}=b w_{1}(z) \\
z\left(t_{i}\right)=z_{i}
\end{array}\right.
$$

225 where the subscript $i$ refers to the "initial" position of the parcel. A set of trajectories, each for a given $226 z_{i}$, is reported in Fig. 2.

$$
M_{y *}=-\left(f_{0} / \beta_{0}\right) w_{*}
$$

236 By using the typical heating/cooling depth $Z / \lambda$ and $(7 \mathrm{~b})$ to evaluate $w_{*}$, one obtains

$$
O\left(w_{*}\right)=\frac{U Z / \lambda}{L} \frac{\beta_{0} L}{f_{0}}=\frac{U Z \beta_{0}}{\lambda f_{0}}
$$

238 Thus, from (42) and (43) the estimate

$$
O\left(M_{y *}\right)=\frac{U Z}{\lambda}
$$


follows. For instance, if $U=10^{-2} \mathrm{~ms}^{-1}, Z=10^{3} \mathrm{~m}, \lambda=10$, equation (44) yields $O\left(M_{y *}\right)=1 \mathrm{~m}^{2} \mathrm{~s}^{-1}$

241 . The latter estimate is hundred times smaller than the transport of the Gulf Stream.

\section{Remark.}

If the quasi-geostrophic dynamics is disregarded, the relationship between the vertical velocity and the thermal forcing, consistent with above results, can be obtained on the basis of a simple twolayer model. Consider two configurations, the former before the action of the thermal forcing and the latter in the presence of it, according to Fig. 3 , left and right panel, respectively.
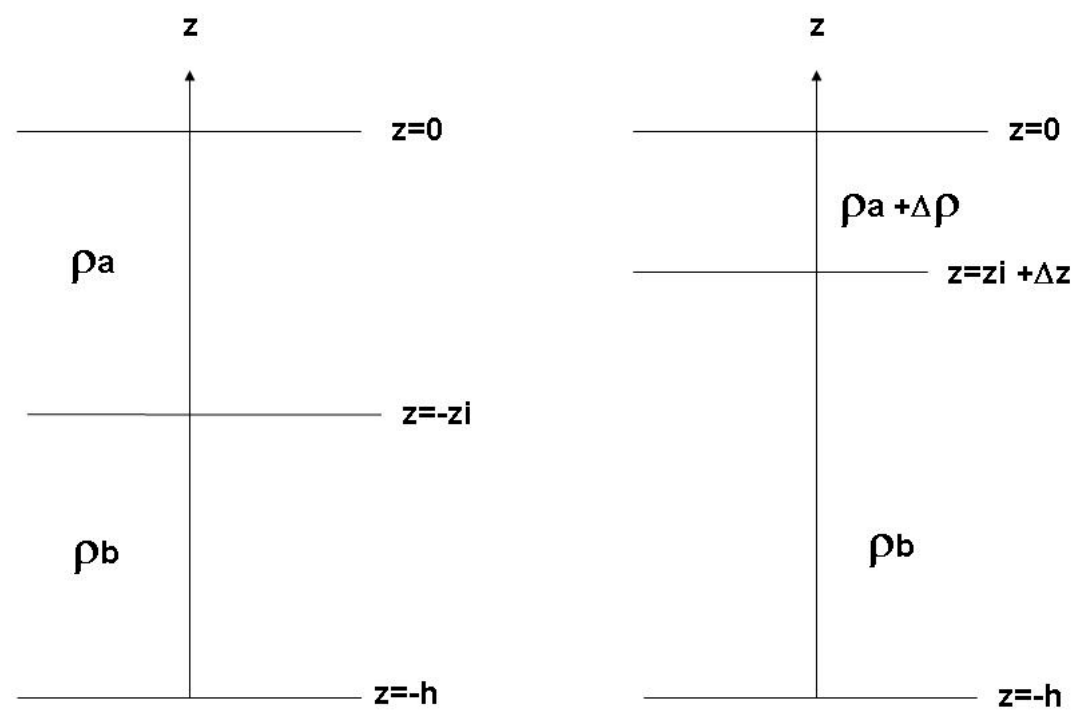

Figure 3. Scheme of the two-layer system to evaluate the vertical velocity of the interface for a varying density of the upper layer.

Mass conservation in a fluid column ${ }^{2}$ implies

$$
z_{i} \rho_{a}+\left(h-z_{i}\right) \rho_{b}=\left(z_{i}-\Delta z\right)\left(\rho_{a}+\Delta \rho\right)+\left(h-z_{i}+\Delta z\right) \rho_{b}
$$

254 and, hence,

$$
z_{i} \Delta \rho+\Delta z\left(\rho_{b}-\rho_{a}-\Delta \rho\right)=0
$$

256 Moreover, conservation of the hydrostatic equilibrium during the process demands

$$
\rho_{b}-\rho_{a}>\Delta \rho
$$

258 Putting $\delta_{\rho}=\rho_{b}-\rho_{a}$, equation (45) gives

${ }^{2}$ Mass conservation is explicitly invoked because of the 1D nature of this model. 


$$
\Delta z=-\frac{z_{i} \Delta \rho}{\delta_{\rho}-\Delta \rho}
$$

Division of (47) by $\Delta t$ and the subsequent application of $\lim _{\Delta t \rightarrow 0}$ yields

$$
w_{i}=\frac{z_{i}}{\delta_{\rho}-\Delta \rho} \frac{\alpha_{\theta} \rho_{0}}{c_{p}} \dot{Q}
$$

262 where $w_{i}=\lim _{\Delta t \rightarrow 0} \frac{\Delta z}{\Delta t}$ and equation (3) has been used. Recalling (46), equation (48) shows that the vertical velocity of the interface is upward for heating and downward for cooling. This is in accordance with the behaviour of $w_{1}$ in the continuum case.

\section{Conclusions}

In the framework of the quasi-geostrophic dynamics at the basin scale, the model of section 4 is probably the simplest and exactly solvable that is able to show how heating affects locally the streamfunction and how the resulting current field arises from the interplay between the thermal forcing and the Sverdrup relation. If the analytic approach is released in favour of a numerical one, the model equations can be made more realistic, although mathematically more complex, on the basis of several generalizations, some of them being listed below:

- The stratification parameter takes explicitly into account its depth dependence;

- The heating is a function of all the coordinates;

- The unperturbed streamfunction is a prescribed function of latitude (not necessarily linear) and of depth.

In doing so one could expect to derive a 3D current field whose validity covers a large portion of the beta-plane, with the obvious exception of the westernmost area.

The transition from an almost conceptual model, like that of section 4, to a numerically solved one introduces another question, that is to say the possibility to detect the phenomenology predicted by the numerical model solution. Because the model refers mainly to the upper ocean, where windwaves and mixing are in permanent activity, the comparison of predictions with field observations might be problematic. This is the case, for instance, of the Ekman's spiral which, on the other hand, can be successfully simulated in a rotating tank. Reasoning in analogy, one could try to resort to a rotating tank experiment to check whether the predicted phenomenology does take place or not. This should be the first step to do. 
289

(A1)

$$
\begin{aligned}
& \frac{D}{D t}\left[\rho_{s}\left(1+r \rho^{\prime}\right)\right]=\left(1+r \rho^{\prime}\right) \frac{D \rho_{s}}{D t}+r \rho_{s} \frac{D \rho^{\prime}}{D t} \approx w \frac{d \rho_{s}}{d z}+r \rho_{s} \frac{U}{L} \frac{D \rho^{\prime}}{D t^{\prime}} \\
& =\frac{U Z}{L} w^{\prime} \frac{d \rho_{s}}{d z}+\frac{U^{2} f_{0}}{g Z} \rho_{s} \frac{D \rho^{\prime}}{D t^{\prime}}=\left(\frac{U Z}{L} \frac{d \rho_{s}}{d z}\right)\left[w^{\prime}+\frac{U L f_{0}}{Z^{2}} \frac{\rho_{s}}{g}\left(\frac{d \rho_{s}}{d z}\right)^{-1} \frac{D \rho^{\prime}}{D t^{\prime}}\right]
\end{aligned}
$$

290 In terms of the Brunt-Vaisala frequency square $N_{s}^{2}=-\frac{g}{\rho_{s}} \frac{d \rho_{s}}{d z}$ one can write

$$
\frac{U L f_{0}}{Z^{2}} \frac{\rho_{s}}{g}\left(\frac{d \rho_{s}}{d z}\right)^{-1}=-\frac{U}{L f_{0}}\left(\frac{L f_{0}}{Z N_{s}}\right)^{2}=-\frac{\varepsilon}{S}
$$

where $\mathcal{E}$ is the Rossby number and $S$ is the stratification parameter. Thus, because of (A2),

293 equation (A1) is equivalent to

294

$$
\frac{D}{D t}\left[\rho_{s}\left(1+r \rho^{\prime}\right)\right]=\left(\frac{U Z}{L} \frac{d \rho_{s}}{d z}\right)\left(w^{\prime}-\frac{\varepsilon}{S} \frac{D \rho^{\prime}}{D t^{\prime}}\right)
$$

295

and, by using (A3), the full equation (3) takes the form

296

$$
w^{\prime}-\frac{\varepsilon}{S} \frac{D \rho^{\prime}}{D t^{\prime}}=-\frac{L}{U Z}\left(\frac{d \rho_{s}}{d z}\right)^{-1} \frac{\rho_{0} \alpha_{\theta}}{c_{p}} \dot{Q}
$$

297 which is conveniently restated as follows

298

(A4)

$$
w^{\prime}=\frac{\varepsilon}{S}\left[\frac{D \rho^{\prime}}{D t^{\prime}}-\frac{S}{\varepsilon} \frac{L}{U Z}\left(\frac{d \rho_{s}}{d z}\right)^{-1} \frac{\rho_{0} \alpha_{\theta}}{c_{p}} \dot{Q}\right]
$$

299 Noting that

300

$$
\frac{S}{\varepsilon} \frac{L}{U Z}\left(\frac{d \rho_{s}}{d z}\right)^{-1} \frac{\rho_{0} \alpha_{\theta}}{c_{p}} \dot{Q}=\frac{L f_{0}}{U} \frac{N_{s}^{2} Z^{2}}{L^{2} f_{0}^{2}} \frac{L}{U Z}\left(\frac{d \rho_{s}}{d z}\right)^{-1} \frac{\rho_{0} \alpha_{\theta}}{c_{p}} \dot{Q}
$$

$$
=\frac{N_{s}^{2} Z}{U^{2} f_{0}}\left(\frac{d \rho_{s}}{d z}\right)^{-1} \frac{\rho_{0} \alpha_{\theta}}{c_{p}} \dot{Q}=-\frac{g Z}{U^{2} f_{0}} \frac{\alpha_{\theta}}{c_{p}} \dot{Q}
$$

301 substitution of (A5) into (A4) gives

302

(A6)

$$
w^{\prime}=\frac{\varepsilon}{S}\left(\frac{D \rho^{\prime}}{D t^{\prime}}+\frac{g Z}{U^{2} f_{0}} \frac{\alpha_{\theta}}{c_{p}} \dot{Q}\right)
$$

303

Finally, by using position

304

(A7)

$$
\frac{g Z \alpha_{\theta} \dot{Q}}{U^{2} f_{0} c_{p}}=\mathrm{H}^{\prime}
$$


305 one ascertains that equation (6) is nothing but (A.6). By using the 1.h.s. of (A7) the order of magnitude 306 of $\mathrm{H}^{\prime}$ can be estimated as follows. Recalling (3), equation (A7) can be restated as $307 \quad \mathrm{H}^{\prime}=-\frac{g Z}{U^{2} f_{0} \rho_{0}} \frac{D \rho}{D t}$ and hence

308

$$
O\left(\mathrm{H}^{\prime}\right)=\frac{g Z}{U^{2} f_{0} \rho_{0}} \frac{\delta \rho}{T}
$$

309 where

$$
\frac{\delta \rho}{T}=O\left(\frac{D \rho}{D t}\right)
$$

311 Reasonable values of the depth of the motion and of the horizontal velocity substituted into (A8) lead 312 to

$313(\mathrm{~A} 10)$

$$
O\left(\mathrm{H}^{\prime}\right)=4 \times 10^{7} \times \frac{\delta \rho}{T}
$$

314 Keeping, for instance, $\delta \rho=0.2 \mathrm{~kg} \mathrm{~m}^{-3}$ and $T=2.6 \times 10^{6} \mathrm{~s}$, that is to say $T=1 \mathrm{month}$, 315 equation (A10) gives

$$
\mathrm{O}\left(\mathrm{H}^{\prime}\right)=1
$$

318 Conflicts of Interest: The authors declare no conflict of interest."

\section{References}

320 [1] M.A. Spall, J. Marine Research, 61, 1-25 (2003)

321 [2] M.A. Spall, J.Phys. Oceanogr., 19, 2341-2347 (2010)

322 [3] Barcilon, V., Pedlosky, J., J.Fluid Mech. 29, 609-621 (1967)

323 [4] J.A. Whitehead, J. Pedlosky, Dynamics of Atmospheres and Oceans, 31, 1-21 (2000)

324 [5] J. Pedlosky, J.Phys. Oceanogr., 33, 2333-2340.(2003)

325 [6] F. Cavallini and F. Crisciani (2013): Quasi-Geostrophic Theory of Oceans and

$326 \quad$ Atmosphere. Springer, 385 pp;

327 [7] J. Pedlosky: Geophysical Fluid Dynamics. Springer, 710 pp (1987)

328 [8] J. Pedlosky: Ocean Circulation Theory. Springer, 453 pp. (1996) 